\title{
Complexity of Epileptiform Activity in a Neuronal Network and Pharmacological Intervention
}

\author{
D. Abásolo ${ }^{1}$, L. González ${ }^{1}$ and Y. Chen ${ }^{2}$ \\ ${ }^{1}$ Centre for Biomedical Engineering, Department of Mechanical Engineering Sciences, Faculty of Engineering and Physical Sciences, \\ University of Surrey, Guildford, UK \\ ${ }^{2}$ Department of Biochemistry and Physiology, Faculty of Health and Medical Sciences, University of Surrey, Guildford, UK
}

\begin{abstract}
Neuronal outputs are complex signals of dynamically integrated excitatory and inhibitory components. Decreased synaptic inhibition in a neuronal network increases excitability and multiple spiking in neurons. Synchronized multiple spiking among a neuronal population further generates rhythmic field potentials and this epileptiform activity can propagate in the brain and cause seizures. Pharmacological interventions that reduce rhythmicity of epileptiform activity may have antiepileptic potentials. We evaluated the LempelZiv (LZ) complexity for identifying rhythmicity in population spikes recorded in granule cells of the murine dentate gyrus in vitro. Blocking synaptic inhibition by the $\mathbf{G A B A}_{\mathrm{A}}$ receptor antagonist, bicuculline, caused epileptiform population spikes, and we found that the $\mathrm{LZ}$ complexity of the signal was significantly reduced. Moreover, the $\mathrm{GABA}_{\mathrm{B}}$ receptor agonist, baclofen, reduced the amplitude of the epileptiform population spike and we found that it increased LZ complexity. The results show that LZ complexity is sensitive to pharmacological interventions that apparently alter rhythmicity of neuronal outputs by desynchronizing neuronal population firing. This novel approach in neuronal signal processing may be used to identify new antiepileptic targets.
\end{abstract}

Keywords - Lempel-Ziv complexity, epileptiform activity, dentate gyrus, excitation, synaptic inhibition

\section{INTRODUCTION}

Epilepsy is one of the most common neurological disorders affecting about $1 \%$ of the population. Paroxysmal neuronal population firing of high amplitude and high rhythmicity is generated in the brain and the epileptiform activity propagates in synchrony causing various forms of seizures. This debilitating condition often requires lifelong treatment. However, causes of seizures are often undiagnosed in individual patient. As treatments are limited to a small number of targets, seizures in as many as $30 \%$ patients are refractory to medication [1]. A full understanding of cellular events and molecular mechanisms that control the occurrence of epileptiform activity is therefore essential for developing more targeted therapeutic approaches.

Increased excitability of neuronal circuits is thought to underlie generation of epileptiform activity. Decreased expression of the inhibitory neurotransmitter GABA (gam- ma-aminobutyric acid) and reduced GABAergic synaptic inhibition are some of the major deficits found in patients and in preclinical animal models [2]. In brain tissue isolated in vitro, blocking $\mathrm{GABA}_{\mathrm{A}}$ receptors significantly reduces synaptic inhibition and neuronal firing pattern is changed from a single spike to repetitive firing following a brief stimulation of excitatory inputs [3]. The repetitive firing is often synchronized within a neuronal population and the resultant rhythmic signals have been predicted to enable widespread synchrony in the brain. Subsequently, pharmacological treatments that reduce rhythmicity of activities may have antiepileptic potentials [4].

In this pilot work the rhythmicity of epileptiform data was studied with Lempel-Ziv (LZ) complexity, a non-linear method of symbolic sequence analysis. We examined population spike (PS) waveforms recorded from granule cells in the dentate gyrus in vitro. Our previous results show that PSs with multiple spikes were induced using $\mathrm{GABA}_{\mathrm{A}}$ receptor antagonist, bicuculline, and this epileptiform activity was reduced in amplitude by the $\mathrm{GABA}_{\mathrm{B}}$ receptor agonist, baclofen [3]. The hypothesis of this study was that LZ complexity would highlight pharmacologically induced changes in rhythms in stimulus evoked PSs.

\section{Material AND METHODS}

\section{A. Data collection}

The PSs were recorded from the soma of granule cells in the dentate gyrus [3]. Briefly, transverse sections of the dentate gyrus and the hippocampus were cut at $300 \mu \mathrm{m}$ thickness using a vibratome. The use of wild-type mice was in accordance with the Animals in Scientific Procedures Act (1986) UK. The brain slices were kept in oxygenated (5\%

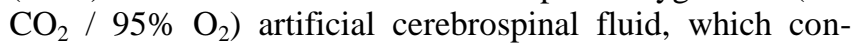
tained (in $\mathrm{mM}$ ) $\mathrm{NaCl}$ (123), $\mathrm{Na}_{2} \mathrm{CO}_{3}$ (25), glucose (10), $\mathrm{KCl}$ (3.7), $\mathrm{CaCl}_{2}$ (2.5), $\mathrm{NaH}_{2} \mathrm{PO}_{4}$ (1.4) and $\mathrm{MgSO}_{4}$ (1.2), and maintained at $31^{\circ} \mathrm{C}$. Recordings of excitatory synaptic potential and PSs were from a multi-electrode probe (MEDP210A; MED64, Alpha MED Sciences, Osaka, Japan), which consists of 64 indium tin oxide and platinum black electrodes arranged in an 8 by 8 grid with an inter-electrode 
distance of $100 \mu \mathrm{m}$. Electrical pulses (0.2 ms in duration) were delivered every $60 \mathrm{~s}$ to stimulate the excitatory inputs, the perforant pathway via one of the electrodes in the array. Stimulation, recording and analysis of extracellular potentials were performed using the Mobius software (version 0.3.7; Alpha MED Sciences, Osaka, Japan).

PS waveforms displayed positively deflected field excitatory synaptic potential superimposed by a large negativelydeflected spike when recorded in the baseline condition. An example of this is shown in Figure 1 (trace identified as B). Following 30 minutes of stable recording of baseline signals, $\mathrm{GABA}_{\mathrm{A}}$ receptor antagonist, (-)-bicuculline (Bic, $10 \mu \mathrm{M}$, Sigma-Aldrich, UK), GABA $A_{B}$ receptor agonist, ( \pm )baclofen (Bac, 10 $\mu \mathrm{M}$, Sigma-Aldrich, UK), and antagonist, CGP55845 (CGP, 1 $\mu \mathrm{M}$, Tocris Bioscience, UK) were examined by bath application for at least 15 minutes to achieve equilibrium. Example PS recordings under each treatment are shown in Figure 1. The PS after drugs washing out is also shown in Figure $1(\mathrm{~W})$.

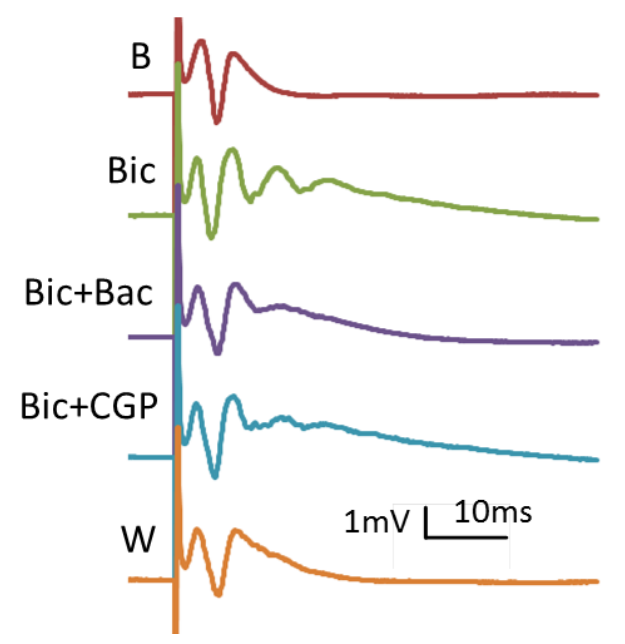

Fig. 1 Example set of PS recordings at baseline (B), and following treatments with bicuculline (Bic), bicuculline and baclofen (Bic+Bac), bicuculline and CGP55845 (Bic+CGP), and after washing out drugs (W). The stimulus artifact is shown at the first $5 \mathrm{~ms}$ of recording.

\section{B. Lempel-Ziv complexity}

LZ complexity is a non-linear method of symbolic sequence analysis. Originally suggested by Lempel and Ziv [5] for data compression, LZ complexity soon found its way for the analysis of complexity in short time series. There are several reasons for this. First of all, LZ complexity is a nonparametric method, i.e. no input parameters have to be defined for its computation. Complexity in the LZ algorithm is related to the number of distinct substrings and the rate of their recurrence along the given sequence [6]. Furthermore, it does not require long data segments to compute [7]. In addition, LZ complexity is model-independent and only the relevant changes between activity patterns that make a difference to the underlying system itself are considered, regardless of the nature - deterministic chaos, stochastic process, etc. - of the system itself [8]. When applying LZ complexity analysis, a particular model of system dynamics is not tested; data differences are examined on the basis of algorithmic or Kolmogorov's complexity (length of the shortest description of a string in some fixed symbolic language) [9].

These useful properties paved the way for the application of LZ complexity to biomedical signal processing. Biomedical signals are often of short length, contaminated by noise and non-stationary. These characteristics make the blind application of classic non-linear methods derived from chaos theory problematic. LZ complexity offers a fast and accurate estimation of complexity in Kolmogorov's sense for biomedical signals.

The LZ complexity algorithm involves converting the original time series into a discrete sequence of a finite number of symbols. In this study the median was used as the threshold $T_{d}$ in the sequence conversion, given that the median is robust to outliers [10]. A sequence $P=s(1), s(2), \ldots$, $s(n)$ is created by comparing the samples from the original sampled signal $x(i)$ with the threshold, with $s(i)$ given by:

$$
s(i)= \begin{cases}0 & \text { if } x(i)<T_{d} \\ 1 & \text { if } x(i) \geq T_{d}\end{cases}
$$

Once this coarse-grained sequence has been created from the original signal, $P$ is scanned from left to right and the complexity counter $c(n)$ is increased by one unit every time a new subsequence of consecutive characters is encountered. A detailed description of the complexity algorithm can be found in [8].

The complexity algorithm is dependent on the sequence length. For this reason, $c(n)$ should be normalized. For a sequence of length $n$ and an alphabet of $\alpha$ symbols (in the case of a binary conversion, $\alpha=2$ ), the upper bound of $c(n)$ is given by [5]:

$$
c(n)<\frac{n}{\left(1-\varepsilon_{n}\right) \log _{\alpha}(n)}
$$

where $\varepsilon_{n}$ is a small quantity and $\varepsilon_{n} \rightarrow 0(n \rightarrow \infty)$. In general,

$$
\lim _{n \rightarrow \infty} c(n)=b(n) \equiv \frac{n}{\log _{\alpha}(n)}
$$

Therefore, $c(n)$ can be normalized via $b(n)$ : 


$$
C(n)=\frac{c(n)}{b(n)}
$$

$C(n)$ is then the normalized LZ complexity. Greater $C(n)$ values correspond to more complexity in the data.

\section{Statistical analysis}

Normality of the LZ complexity results was evaluated using Lilliefors test. Treatment effects were compared using repeated-measures one-way ANOVA followed by Tukey's multiple comparisons. Statistical significance was taken as $p<0.05$. All statistical analyses were performed using Prism 5 (GraphPad Software Inc. La Jolla, CA 92037 USA).

\section{RESULTS}

LZ complexity was estimated from six datasets of PS waveforms recorded with a sampling frequency of $20 \mathrm{kHz}$ under the 5 different conditions: baseline, bicuculline, bicuculline and baclofen, bicuculline and CGP55845, and after drugs washing out. Thirty minutes of stable recording was usually carried out before any drug treatment during the baseline period (B, Figure 1). To induce epileptiform activity, bicuculline (Bic, $10 \mu \mathrm{M})$ was applied to the brain slice for 15 minutes (Bic, Figure 1). Multiple spiking PSs were recorded due to repetitive firing in individual neurons, reflecting increased excitability. Then, baclofen $(10 \mu \mathrm{M})$ was added to the bicuculline medium (Bic+Bac, Figure 1) to see if $\mathrm{GABA}_{\mathrm{B}}$ receptor activation can affect epileptiform activity. Significant reduction in the amplitude of late PSs was found [3], but whether the rhythm persisted or not was unclear. To determine whether the anti-epileptiform effect of baclofen is due to receptor activation, we also examined CGP55845 in the presence of bicuculline (Bic+CGP, Figure 1 ), and an increase in late PSs was observed. Finally, washout of both bicuculline and CGP55845 (W, Figure 1) restored the PS to the single spike waveform at baseline. The PS waveforms at these conditions show distinct characteristics in rhythm, so that LZ complexity analysis was performed and results are shown in Table 1. The stimulus artifact at $5 \mathrm{~ms}$ of each recording was not included in the analysis.

LZ complexity was significantly reduced in bicucullinetreated PS waveforms in all data sets from the baseline ( $p<0.01$, B vs. Bic, Table 1), potentially reflecting increased rhythmicity in epileptiform activity. Addition of baclofen to bicuculline increased LZ complexity values significantly $(p<0.05$, Bic vs. Bic+Bac, Table 1) to a level that is not different from the baseline $(p>0.05$, Bic+Bac vs. B, Table 1). $G_{A B} A_{B}$ receptor activation therefore reduced rhythmicity in the epileptiform activity. Blocking $\mathrm{GABA}_{\mathrm{B}}$ receptors with CGP55845, moreover, reduced the complexity $(p<0.01$, Bic + Bac vs. Bic+CGP, Table 1$)$ back down again to a level similar to bicuculline alone $(p>0.05$, Bic+CGP vs. Bic, Table 1). It is also noted that the co-treatment of bicuculline and CGP55845 produced more consistently lower LZ complexity that is also significantly different from the baseline $(p<0.001$, Bic+CGP vs. B, Table 1$)$ and washout $(p<0.001$, Bic+CGP vs. W, Table 1). Finally, returning to drug-free medium effectively increased LZ complexity $(p<0.001, \mathrm{Bic}+\mathrm{CGP}$ vs. W, and $p<0.01, \mathrm{Bic}$ vs. W, Table 1$)$ to the level of the baseline ( $p>0.05$, B vs. W, Table 1$)$.

Table $1 \mathrm{LZ}$ complexity results for six datasets of PS waveforms under different pharmacological treatment conditions

\begin{tabular}{|c|c|c|c|c|c|}
\hline Set & B & Bic & $\mathrm{Bic}+\mathrm{Bac}$ & $\mathrm{Bic}+\mathrm{CGP}$ & $\mathrm{W}$ \\
\hline 1 & 0.1750 & 0.0255 & 0.1640 & 0.0292 & 0.2078 \\
\hline 2 & 0.1130 & 0.0693 & 0.0765 & 0.0838 & 0.1349 \\
\hline 3 & 0.1203 & 0.0365 & 0.0729 & 0.0328 & 0.0620 \\
\hline 4 & 0.2151 & 0.0401 & 0.2406 & 0.0547 & 0.2624 \\
\hline 5 & 0.1932 & 0.1567 & 0.1859 & 0.0911 & 0.1859 \\
\hline 6 & 0.2880 & 0.1349 & 0.2114 & 0.0875 & 0.2187 \\
\hline $\begin{array}{ll}\text { Mean } \pm \\
\text { SD }\end{array}$ & $\begin{array}{c}0.1841 \pm \\
0.0649\end{array}$ & $\begin{array}{c}0.0772 \pm \\
0.0555\end{array}$ & $\begin{array}{c}0.1586 \pm \\
0.0698\end{array}$ & $\begin{array}{c}0.0632 \pm \\
0.0281\end{array}$ & $\begin{array}{c}0.1786 \pm \\
0.0708\end{array}$ \\
\hline
\end{tabular}

B: baseline period before any treatment; Bic: treatment with bicuculline $(10 \mu \mathrm{M})$; Bic+Bac: co-application of bicuculline $(10 \mu \mathrm{M})$ and baclofen $(10 \mu \mathrm{M})$; Bic+CGP: co-application of bicuculline $(10 \mu \mathrm{M})$ and CGP55845 $(1 \mu \mathrm{M})$; W: washout of drugs.

\section{DisCUSSION AND CONCLUSIONS}

The results show that LZ complexity of PS waveforms changes significantly following pharmacological treatments. Firstly, the LZ complexity algorithm has identified significantly reduced complexity following bicuculline treatment compared to the baseline control waveform. The $\mathrm{GABA}_{\mathrm{A}}$ receptor antagonist, bicuculline, blocks synaptic inhibition that suppresses recurrent excitation and reduces excitability of neurons [2]. As a result, multiple spiking in burst occurs in neurons. Synchronization among a group of neurons gives rise to multiple spikes at regular intervals in the population field potential and rhythmicity is increased. Conversely, the baseline PS waveforms with single spike show higher levels of complexity, as the signal is the result of complex integration of excitatory and inhibitory actions. Therefore, reduced LZ complexity is found in epileptiform activity associated with increased rhythmicity due to synchronous multiple spiking in neurons.

$\mathrm{GABA}_{\mathrm{B}}$ receptors are G-protein coupled receptors for the inhibitory neurotransmitter GABA. They mediate inhibitory actions in the brain by presynaptic inhibition of neurotransmitters and postsynaptic inhibition of membrane excitability [11]. The receptor agonist, baclofen, has been reported to suppress epileptiform activity and seizures in patients and in 
animal models of seizures, showing an antiepileptic action [12]. In the dentate gyrus, $\mathrm{GABA}_{\mathrm{B}}$ receptors are predominantly expressed on inhibitory pathways to modulate GABA release presynaptically, and on granule cells to regulate their excitability postsynaptically. When $\mathrm{GABA}_{\mathrm{A}}$ receptors are blocked by bicuculline, the effect of baclofen on granule cells is more likely to be postsynaptic, and this reduced amplitude of the epileptiform activity. Now we show that LZ complexity is significantly increased by baclofen, so that rhythmicity in the epileptiform activity is reduced, indicating desynchronization between neurons. $\mathrm{GABA}_{\mathrm{B}}$ receptor activation in the dentate gyrus is therefore potentially antiepileptic by reducing rhythmicity in epileptiform activity.

Accordingly, GABA $A_{B}$ receptor antagonist, CGP55845, significantly reduced LZ complexity to the level of bicuculline, opposite to the effect of baclofen. Reduction in rhythmicity is therefore specific to activation of $\mathrm{GABA}_{B}$ receptors. Moreover, the co-treatment of bicuculline and CGP55845 consistently produced lower LZ complexity, indicating additional rhythmic effect of $\mathrm{GABA}_{\mathrm{B}}$ receptor blockade.

Finally, washing out of both bicuculline and CGP55845 restored both the baseline waveform of PSs and LZ complexity. The LZ complexity analysis can therefore distinguish the different pharmacological treatments, indicating its potential for measuring rhythmicity in epileptiform activity.

To the best of our knowledge, this is the first time LZ complexity has been successfully applied to the detection of significant changes induced pharmacologically in short, stimulus-evoked traces with limited number of repetitive cycles. It is, however, noted that the LZ complexity values obtained were scattered with considerable overlap between treatments. For example, some epileptiform traces have higher complexity values than baseline traces in other sets. However, within each dataset the trend is consistent. Further investigation is required to look into the potentially confounding characteristics in these traces. In addition, signal averaging may be applied to further eliminate contamination by rhythmic noises. Furthermore, although results are promising, the sample size was small. As a result, our findings are preliminary and require replication in a larger database of PS waveforms before any conclusion can be made of its potential impact. However, our findings show that the non-linear analysis of stimulus evoked-traces identifies pharmacologically induced changes.

In summary, we found that LZ complexity was significantly reduced in bicuculline-induced epileptiform activity and baclofen restored the reduced LZ complexity to the control level. LZ complexity may therefore be used as a novel analysis to measure rhythmicity in epileptiform activity. Caution should be applied due to the small sample size.

\section{ACKNOWLEDGMENT}

This work was supported by the Engineering and Physical Sciences Research Council (EPSRC) [grant number EP/I000992/1] to Ying Chen and Daniel Abásolo, and BBSRC [grant number BB/E010296/1] to Ying Chen. We thank Joshua Foster for data collection.

\section{REFERENCES}

1. Romanelli P, Striano P, Barbarisi M et al. (2012) Non-resective surgery and radiosurgery for treatment of drug-resistant epilepsy. Epilepsy Res 99:193-201 DOI 10.1016/j.eplepsyres.2011.12.016

2. Scharfman HE (2007) The Neurobiology of epilepsy. Curr Neurol Neurosci Rep 7:348-354 10.1007/s11910-007-0053-z

3. Foster JD, Kitchen I, Bettler B et al. (2013) $\mathrm{GABA}_{\mathrm{B}}$ receptor subtypes differentially modulate synaptic inhibition in the dentate gyrus to enhance granule cell output. Br J Pharmacol 168:1808-1819 DOI $10.1111 / \mathrm{bph} .12073$

4. Löscher W (2011) Critical review of current animal models of seizures and epilepsy used in the discovery and development of new an$\begin{array}{llll}\text { tiepileptic } & \text { drugs. } & \text { Seizure } 359-368 & \text { DOI }\end{array}$ 10.1016/j.seizure.2011.01.003

5. Lempel A, Ziv J (1976) On the complexity of finite sequences. IEEE Trans Inform Theory 22:75-81 DOI 10.1109/TIT.1976.1055501

6. Radhakrishnan N, Gangadhar BN (1998) Estimating regularity in epileptic seizure time-series data. A complexity-measure approach. IEEE Eng Med Biol 17:89-94 DOI 10.1109/51.677174

7. Zhang XS, Zhu YS, Thakor NV et al. (1999) Detecting ventricular tachycardia and fibrillation by complexity measure. IEEE Trans Biomed Eng 46:548-555 DOI 10.1109/10.759055

8. Zhang XS, Roy RJ, Jensen EW (2001) EEG complexity as a measure of depth of anesthesia for patients. IEEE Trans Biomed Eng 48:14241433 DOI 10.1109/10.966601

9. Kolmogorov AN (1965) Three approaches to the quantitative definition of information. Infor Trans 1:3-11

10. Nagarajan R (2002) Quantifying physiological data with Lempel-Ziv complexity - Certain issues. IEEE Trans Biomed Eng 49:1371-1373 DOI 10.1109/TBME.2002.804582

11. Bettler B, Kaupmann K, Mosbacher J et al. (2004) Molecular structure and physiological functions of GABA(B) receptors. Physiol Rev 84:835-867 DOI 10.1152/physrev.00036.2003

12. Chandler KE, Princivalle AP, Fabian-Fine R et al. (2003). Plasticity of $\mathrm{GABA}(\mathrm{B})$ receptor-mediated heterosynaptic interactions at mossy fibers after status epilepticus. J Neurosci 23: 11382-11391

\author{
Author: Daniel Abásolo \\ Institute: University of Surrey \\ City: Guildford \\ Country: United Kingdom \\ Email: d.abasolo@surrey.ac.uk
}

